\title{
Research on High Efficiency Production of Enterprise Based on Fuzzy Comprehensive Evaluation Model
}

\author{
Jingjing Feng, ${ }^{1, *}$ \\ ${ }^{1}$ School of Intelligent Science and Information Engineering, Xi'an Peihua University, Xi'an, Shaanxi 710125, China \\ *Corresponding author. Email: fengjingjing@ @eihua.edu.cn
}

\begin{abstract}
In the information age, the fuzziness of things urgently requires mathematics and quantification. First of all, taking clothing as an example, this paper introduces the first level comprehensive evaluation model, and uses this model to judge customers' preferences for clothing, so as to organize production and goods. On this basis, the one-level comprehensive evaluation model is extended to the multi-level evaluation model. Taking product quality detection as an example, it can be seen that the multi-level comprehensive evaluation model can better detect product quality. Finally, the application scope of the one-level comprehensive evaluation model and the multi-level comprehensive evaluation model are obtained.
\end{abstract}

Keywords: fuzzy, one-level model, multi-level model, comprehensive evaluation

\section{INTRODUCTION}

Fuzzy phenomena and fuzzy concepts are everywhere, such as "young people", "tall people", "high-quality products", "healthy people". These concepts formed in human brain are almost fuzzy. We can think that fuzzy phenomena are an essential thing for human beings, especially with the coming of information age, the contradiction between accuracy and fuzziness is more prominent. If people want to study the essence of fuzzy phenomenon, they need to make it mathematical and quantitative. Fuzzy mathematics is a branch of mathematics to solve this fuzzy phenomenon. Fuzzy comprehensive evaluation method is a comprehensive evaluation method based on fuzzy mathematics. Based on the membership theory of fuzzy mathematics, qualitative evaluation is transformed into quantitative evaluation, that is, fuzzy mathematics is used to make overall evaluation of things or objects restricted by many factors. The evaluation result of this method is clear and systematic, which can solve the fuzzy and difficult to quantify problems and is suitable for the uncertain problems.

Fuzzy comprehensive evaluation method has been widely used. For example, fuzzy comprehensive evaluation method has been applied to product design[1,2], benefit comprehensive evaluation[3-5], risk evaluation[7] and other aspects. This article introduces the one-level comprehensive evaluation model, and uses this model to judge customers' preferences for clothing. And then the first level comprehensive evaluation model is extended to the multi-level evaluation model. Taking product quality detection as an example, it can be seen that the multi-level comprehensive evaluation model can better detect product quality.

\section{FIRST LEVEL COMPPREHENSIVE EVALUATION MODEL}

\subsection{The one-level mode}

According to the determined standard, evaluating a factor or a part of an object or a class of objects is called single evaluation. It is called comprehensive evaluation to get the overall evaluation of a certain kind of object from many single evaluations. The purpose of comprehensive evaluation is to sort some objects in a certain sense and select the best and the worst objects, which is also called decision-making process.

The steps of using the one-level model for comprehensive evaluation are as follows:

(1) Determine the factor set of evaluation object

$U=\left\{u_{1}, u_{2}, \cdots, u_{n}\right\}$.

(2) Give judgment set $V=\left\{v_{1}, v_{2}, \cdots, v_{n}\right\}$.

(3) Single factor evaluation, that is to establish a fuzzy mapping from $U$ to $V, \tilde{f}: U \rightarrow F(V)$, $x_{i} \mapsto \frac{r_{i 1}}{v_{1}}+\frac{r_{i 2}}{v_{2}}+\cdots+\frac{r_{i m}}{v_{m}}$, in which $0 \leq r_{i j} \leq 1$, $i=1,2, \cdots, m$. Deriving fuzzy relation $R_{\tilde{f}}$ from $\tilde{f}$, expressed by matrix $R=\left(\begin{array}{cccc}r_{11} & r_{12} & \cdots & r_{1 m} \\ r_{21} & r_{22} & \cdots & r_{2 m} \\ \vdots & \vdots & & \vdots \\ r_{n 1} & r_{n 2} & \cdots & r_{n m}\end{array}\right)$.

$R$ is called single factor evaluation matrix. 
(4) Comprehensive evaluation.

\subsection{Apparel evaluation}

Take clothing evaluation as an example. Suppose that when a customer buys a certain garment, the factor set

$U=\left\{u_{1}, u_{2}, u_{3}\right\}$, among them, $u_{1}, u_{2}, u_{3}$ represent pattern, durability and price cost respectively. If judging clothing, it can be recorded as $V=\left\{v_{1}, v_{2}, v_{3}, v_{4}\right\}$, among them,

$v_{1}, v_{2}, v_{3}, v_{4}$ represent very like, like, litter like and dislike respectively. Single factor evaluation is to invite a number of professionals or customers to express their opinions on the design, durability and price. In terms of decors, if 70 percent of people very like it, 20 percent of people like it, 10 percent of people litter like it. That is, $x_{1} \mapsto(0.7,0.2,0.1,0)$.Similarly, if we have

$x_{2} \mapsto(0.2,0.4,0.3,0.1), x_{3} \mapsto(0.1,0.3,0.4,0.2)$, then there is a single factor evaluation matrix

$$
R=\left(\begin{array}{cccc}
0.7 & 0.2 & 0.1 & 0 \\
0.2 & 0.4 & 0.3 & 0.1 \\
0.1 & 0.3 & 0.4 & 0.2
\end{array}\right) .
$$

When customers look at this kind of clothes, they are happy to decide whether to buy them or to hesitate, or to be indifferent and abandon them, which can be seen as the result of customers' comprehensive balance of design, style, durability and price. All kinds of customers have different "focus", which is the basis for their respective comprehensive trade-offs. This "focus" can be quantified. If a customer's attention to design and style is $50 \%$, to durability is $30 \%$, and to price is $20 \%$,then

$\tilde{A}=(0.5,0.3,0.2)$. Obviously, $\tilde{A}$ is the vector representation of a fuzzy subset of factor set $X$, which is usually called the weight set in comprehensive evaluation.

So what is the attitude of weight $\tilde{A}$ customers towards this kind of clothing? This is a process of comprehensive evaluation, that is, the fuzzy transformation from $U$ to $V$.

$$
\begin{aligned}
& \tilde{B}=\tilde{A} \circ R=\left(\begin{array}{lll}
0.5 & 0.3 & 0.2
\end{array}\right)\left(\begin{array}{cccc}
0.7 & 0.2 & 0.1 & 0 \\
0.2 & 0.4 & 0.3 & 0.1 \\
0.1 & 0.3 & 0.4 & 0.2
\end{array}\right) \\
& =(0.5,0.3,0.3,0.2)
\end{aligned}
$$

Normalization

$$
\left(\frac{0.5}{1.3}, \frac{0.3}{1.3}, \frac{0.3}{1.3}, \frac{0.2}{1.3}\right) \approx(0.38,0.25,0.25,0.12) .
$$

It means that the customers with weight $\tilde{A}$ evaluate this kind of clothing as follows: the "very like " level is $38 \%$, the " like " level and the " litter like " level are both 25\%, the "dislike" level is $12 \%$.
According to the principle of maximum subordination, the conclusion is "I like it very much".This process is called the positive problem of comprehensive evaluation. If the result is known and the reason is asked, it is the inverse problem of comprehensive evaluation. Taking clothing as an example, if a shopping mall purchases a kind of clothing, which is very popular in the market, then the shopping mall and the production unit should analyze the reasons for the best-selling together. Only by making clear of it, can they organize the production and organization of goods, and the important one is what the weight of customers is. The inverse problem of comprehensive evaluation is the inverse problem of fuzzy transformation, and its solution is the problem of solving fuzzy relation.

\section{MULTI-LEVEL MODEL}

\subsection{Shorts of the $M(\wedge, \vee)$ model}

In the comprehensive evaluation, model $M(\wedge, \vee)$ is used to calculate $B=\tilde{A} \circ R$, among which $b_{j}=\vee_{i=1}^{n}\left(a_{i} \wedge r_{i j}\right)$ $(j=1,2, \cdots, m)$. Operators $(\wedge, \vee)$ have good algebraic properties, but they also have defects. When there are many factors to be considered and $\sum_{i=1}^{n} a_{i}=1$ is required, the weight of each factor $a_{i}$ is so small that $a_{i} \leq r_{i j}$. Because of $b_{j}=\vee_{i=1}^{n}\left(a_{i} \wedge r_{i j}\right)$, a lot information of $R=\left(r_{i j}\right)$ are lost:

$u_{i} \mapsto \tilde{f}\left(u_{i}\right)=\frac{r_{i 1}}{v_{1}}+\frac{r_{i 2}}{v_{2}}+\cdots+\frac{r_{i m}}{v_{m}}=\left(r_{i 1}, r_{i 2}, \cdots, r_{i m}\right)$

That is, people's judgment information about each factor $u_{i}$ is not fully utilized. Therefore, it is often difficult to distinguish the results of comprehensive evaluation decision.

For example, the quality of a product produced by an enterprise is determined by nine indicators $u_{1}, u_{2}, \cdots, u_{9}$, and the product level is divided into four levels: first level, second level, third level and waste. So factor set $U=\left\{u_{1}, u_{2}, \cdots, u_{9}\right\}$, judgement set $V=\left\{v_{1}, v_{2}, v_{3}, v_{4}\right\}$, which $v_{1}, v_{2}, v_{3}, v_{4}$ represent first level, second level, third level and waste respectively. Ask relevant experts, quality inspectors and users to form a single factor evaluation group (scoring or voting). The single factor evaluation matrix is 


$$
\mathrm{R}=\left(\begin{array}{llll}
0.36 & 0.24 & 0.13 & 0.27 \\
0.20 & 0.32 & 0.25 & 0.23 \\
0.40 & 0.22 & 0.26 & 0.12 \\
0.30 & 0.28 & 0.24 & 0.18 \\
0.26 & 0.36 & 0.12 & 0.20 \\
0.22 & 0.42 & 0.16 & 0.10 \\
0.38 & 0.24 & 0.08 & 0.20 \\
0.34 & 0.25 & 0.30 & 0.11 \\
0.24 & 0.28 & 0.30 & 0.18
\end{array}\right)
$$

Set the weight distribution as

$$
\tilde{A}=(0.1,0.12,0.07,0.07,0.16,0.1,0.1,0.1,0.18),
$$

then we have

$\widetilde{B}=\widetilde{A} \circ R=(0.18,0.18,0.18,0.18,0.18)$ by model

$M(\wedge, \vee)$. The result shows that $M(\wedge, \vee)$ is defective and needs to be improved.

\subsection{Multi-level model}

In practical problems, when there are many factors and the weight distribution is relatively uniform, the multi-level model can be adopted. This paper mainly introduces two levels of model - the two-level model.

The steps of using the two-level model for comprehensive evaluation are as follows:

(1) Divide factor set $U=\left\{u_{1}, u_{2}, \cdots u_{n}\right\}$ into several groups $U=\left\{U_{1}, U_{2}, \cdots, U_{k}\right\}$, such that $U=\bigcup_{i=1}^{k} U_{i}$,

$U_{i} \cap U_{j}=\Phi \quad(i \neq j), U=\left\{U_{1}, U_{2}, \cdots, U_{k}\right\}$ is called the first factor set.

Let $U_{i}=\left\{u_{1}^{(i)}, u_{2}^{(i)}, \cdots, u_{n_{i}}^{(i)}\right\}(i=1,2, \cdots, k) \quad$,which $n_{1}+n_{2}+\cdots n_{k}=\sum_{i=1}^{k} n_{i}=n$, then $U_{i}$ is called the second factor set.

(2) Let $V=\left\{v_{1}, v_{2}, \cdots, v_{m}\right\}$ be a evaluation set. First, single factor evaluation is made on the $n_{i}$ factors of the second level factor set $U_{i}=\left\{u_{1}^{(i)}, u_{2}^{(i)}, \cdots, u_{n_{i}}^{(i)}\right\}$, that is, the fuzzy mapping $\tilde{f}_{i}: U_{i} \mapsto F(V)$ is needed.

$$
\begin{aligned}
& u_{1}^{(i)} \mapsto \tilde{f}_{i}\left(u_{1}^{(i)}\right)=\left(r_{11}^{(i)}, r_{12}^{(i)}, \cdots, r_{1 m}^{(i)}\right), \\
& u_{2}^{(i)} \mapsto \tilde{f}_{i}\left(u_{2}^{(i)}\right)=\left(r_{21}^{(i)}, r_{22}^{(i)}, \cdots, r_{2 m}^{(i)}\right),
\end{aligned}
$$

$$
u_{n_{i}}^{(i)} \mapsto \tilde{f}_{i}\left(u_{n_{i}}^{(i)}\right)=\left(r_{n_{i} 1}^{(i)}, r_{n_{i} 2}^{(i)}, \cdots, r_{n_{i} m}^{(i)}\right)
$$

We obtained that the single factor evaluation matrix

$$
R_{i}=\left(\begin{array}{cccc}
r_{11}^{(i)} & r_{12}^{(i)} & \cdots & r_{1 m}^{(i)} \\
r_{21}^{(i)} & r_{22}^{(i)} & \cdots & r_{2 m}^{(i)} \\
\cdots & \cdots & \cdots & \cdots \\
r_{n_{i} 1}^{(i)} & r_{n_{i} 2}^{(i)} & \cdots & r_{n_{i} m}^{(i)}
\end{array}\right)_{n_{i} \times m} .
$$

Let the weight of $U_{i}=\left\{u_{1}^{(i)}, u_{2}^{(i)}, \cdots, u_{n_{i}}^{(i)}\right)$ is $\tilde{A}_{i}=\left(a_{1}^{(i)}, a_{2}^{(i)}, \cdots, a_{n_{i}}^{(i)}\right)$, then we obtained that the onelevel comprehensive evaluation $\tilde{A}_{i} \circ R_{i}=\widetilde{B}_{i}(i=1,2, \cdots, m)$. (3) Treat each $U_{i}$ as an element, use $\tilde{B}_{i}$ as its single factor evaluation, and then get the single factor evaluation matrix

$$
R=\left(\begin{array}{c}
\widetilde{B}_{1} \\
\widetilde{B}_{2} \\
\vdots \\
\tilde{B}_{k}
\end{array}\right)
$$

Let the weight of $U=\left(U_{1}, U_{2}, \cdots, U_{k}\right)$ be $\tilde{A}=\left(a_{1}, a_{2}, \cdots, a_{k}\right)$, then there is a two-level comprehensive evaluation $\tilde{B}=\tilde{A} \circ R$.

Continue to consider that an enterprise produces a product whose quality is determined by nine indicators that $u_{1}, u_{2}, \cdots, u_{9}$.The product level is divided into four levels: first, second, third and waste. Because there are many factors and the weight distribution is relatively balanced, the two-level model should be adopted.

(1) Divide the factor $\operatorname{set} U=\left\{u_{1}, u_{2}, \cdots, u_{9}\right\}$ into three groups:

$U_{1}=\left\{u_{1}, u_{2}, u_{3}\right\}, U_{2}=\left\{u_{4}, u_{5}, u_{6}\right\}, U_{3}=\left\{u_{7}, u_{8}, u_{9}\right\}$

(2) Let judgement $\operatorname{set} V=\left\{u_{1}, u_{2}, u_{3}, u_{4}\right\}, u_{1}$ : the first level, $u_{2}$ : the second level, $u_{3}$ : the third level, $u_{4}:$ waste.

For single factor evaluation of each factor in $U_{i}(i=1,2,3)$, relevant experts, quality inspectors and users can be invited to form an evaluation group, and the corresponding single factor evaluation matrix can be obtained by scoring or voting.

For the second level factor set $U_{i}(i=1,2,3)$, we have $U_{1}=\left\{u_{1}, u_{2}, u_{3}\right\}$, which the weight is $\tilde{A}_{1}=(0.3,0.42,0.28)$, and the single factor evaluation matrix is 


\section{ACKNOWLEDGMENT}

$$
R_{1}=\left(\begin{array}{llll}
0.36 & 0.24 & 0.13 & 0.27 \\
0.20 & 0.32 & 0.25 & 0.23 \\
0.40 & 0.22 & 0.26 & 0.12
\end{array}\right)
$$

Make a comprehensive evaluation at the first level, we have $\widetilde{B}_{1}=\widetilde{A}_{1} \circ R_{1}=(0.3,0.32,0.26,0.27)$ by $M(\wedge, \vee)$. Similarly, we have $U_{2}=\left\{u_{4}, u_{5}, u_{6}\right\}$, which the weight is $\tilde{A}_{2}=(0.2,0.5,0.3)$, and the single factor evaluation matrix is $R_{2}=\left(\begin{array}{llll}0.30 & 0.28 & 0.24 & 0.18 \\ 0.26 & 0.36 & 0.12 & 0.20 \\ 0.22 & 0.42 & 0.16 & 0.10\end{array}\right)$. Then we obtained $\widetilde{B}_{2}=\widetilde{A}_{2} \circ R_{2}=(0.26,0.36,0.2,0.2)$. we have $U_{3}=\left\{u_{7}, u_{8}, u_{9}\right\}$, which the weight is $\widetilde{A}_{3}=(0.3,0.3,0.4)$, and the single factor evaluation matrix is $R_{3}=\left(\begin{array}{cccc}0.38 & 0.24 & 0.08 & 0.20 \\ 0.34 & 0.25 & 0.30 & 0.11 \\ 0.4 & 0.28 & 0.30 & 0.18\end{array}\right)$. Then we obtained $\widetilde{B}_{3}=\widetilde{A}_{3} \circ R_{3}=(0.3,0.28,0.3,0.2)$.

(2) For the first level factor set $U=\left\{U_{1}, U_{2}, U_{3}\right\}$, let the weight distribution as $\tilde{A}=(0.2,0.35,0.45)$, and let the total single factor evaluation matrix be

$$
R=\left(\begin{array}{c}
\tilde{B}_{1} \\
\tilde{B}_{2} \\
\vdots \\
\tilde{B}_{k}
\end{array}\right)=\left(\begin{array}{cccc}
0.3 & 032 & 026 & 0.27 \\
0.26 & 0.36 & 0.2 & 0.2 \\
0.3 & 0.28 & 0.3 & 0.2
\end{array}\right) .
$$

By making a two-level comprehensive evaluation, we can get $\tilde{B}=\tilde{A} \circ R=(0.3,0.35,0.3,0.2)$.

According to the principle of maximum subordination, this product belongs to the second grade.

\section{CONCLUSION}

In the comprehensive evaluation, when there are not many factors to consider, we can use the first level comprehensive evaluation model to select the best and the worst objects and make decisions. But in practical problems, we need to consider many factors. When we meet a lot of factors and the weight distribution is relatively uniform, the results of comprehensive evaluation and decision-making are often difficult to distinguish. At this time, we can use multi-level model for comprehensive evaluation.
This work was supported by the Special Scientific Research Project of Shaanxi Provincial Department of Education(19JK0635).

\section{REFERENCES}

[1] Dongmei Yang, Jiannan Zhang, Xiaoyun Xu, Design of elderly shopping cart based on fuzzy comprehensive evalution method,Journal of Machine Design, 2016, pp:117-120.DOI:https://doi.org/ 10.13841/j.cnki.jxsj.2016.09.025

[2] Shan Hu, Jing Liu, Application of fuzzy comprehensive evaluation method in product design scheme decision, Journal of Machine Design,2020, pp:135-139.

DOI: https://doi.org/ 10.13841/j.cnki.jxsj.2020.01.024

[3] Li Xu, Xinyi Wang, Xuesong Guan, Application of fuzzy comprehensive evaluation in green express packaging, Packaging Engineering,2019, pp:180-188. DOI: https:// doi.org/10.19554/j.cnki.10013563.2019.23.027

[4] Junyi Chen, Qinhong Wu, Ping Yang, Study on fuzzy comprehensive evaluation of PPP project performance of zigong municipal solid waste incineration power plant based on entropy method, Journal of Engineering Management,2020,pp:92-96. DOI: https:// doi.org/10.13991/j.cnki.jem.2020.01.017

[5] Xiaomei Qu, Kang Fu, Qingchun Long, Research on benefit evaluation of power demand side management based on improved fuzzy analytic hierarchy process, Journal of Hubei Normal University (Natural Science), 2019,pp:1-9.DOI:http://doi.org/10.3969/j.issn.20963149.2019.04.001

[6] Saiyan Yan, Research on the financing effect of technology-based SMEs based on AHP fuzzy mathematics comprehensive evaluation, Journal of Industrial Technological Economics, 2020, pp:75-81. DOI: $\quad$ https://doi.org/ 10.3969/j.issn.1004910X.2020.03.009

[7] Hongchun Wang, Hongyun Liu, Dandan Wu, Research on risk assessment of assembly building supply chain based on multi - level fuzzy evaluation method, Journal of Beijing University of Civil Engineering and Architecture, 2019, pp:83-88. DOI: http://doi.org/ 10.19740/j.1004-6011.2019.04.12 